Ein Regionales Entwicklungskonzept für die Region Flensburg/Schleswig

\title{
Nachhaltigkeit des Impulses sichern
}

\begin{abstract}
Anfang 1997 beauftragte die Wirtschaftsförderungs- und Regionalentwicklungsgesellschaft Flensburg/Schleswig mbH (WiREG) das Instifut für ökologische Wirtschaftsforschung, ein integrierfes Regionales Entwicklungskonzept für die Region Flensburg/Schleswig zu erstellen. Im Zuge eines zweijährigen konsultativen Verfahrens bearbeifete das IÖW gemeinsam mif den regionalen Akfeuren regionsspezifische Handlungsfelder (1). Dabei wurden zahlreiche Potentiale aufgedeckt, die durch eine institutionelle Unterstützung daverhaft erschlossen werden könnten.
\end{abstract}

Von Susanne Wittek

$\mathrm{D}$ as Regionale Entwicklungskonzept (REK) ist ein Instrument dezentraler, kooperativer Regionalplanung, der die Selbstverantwortung der regionalen Handlungsträger als Bedingung einer eigenständigen Regionalentwicklung gilt. Die Beteiligung der Akteure am Willensbildungs- und Gestaltungsprozeß bildet den Kern des REK, das als rechtlich nicht verbindliches Konzept in seiner Umsetzung von der Selbstbindung der beteiligten Handlungsträger lebt. Bestimmendes Moment ist die Partizipation der Betroffenen an dezentralisierten Entscheidungsabläufen. Dabei spielen persönliche Kontakte zwischen verschiedenen Handlungsträgern aus politischen, ökonomischen und sozialen Netzwerken eine entscheidende Rolle für die regionale Innovationsfähigkeit.

Die Erstellung des REK für die Region Flensburg/Schleswig wurde mit Mitteln des schleswigholsteinischen Regionalprogramms für strukturschwache ländliche Räume gefördert, das auf die Verbesserung der Beschäftigungs- und Qualifizierungschancen bei gleichzeitigem Schutz der ökologischen Funktion des Raumes zielt (2). Hintergrund war die zunehmende Orientierung von strukturpolitischen Landes-, Bundesund EU-Programmen, vorrangig solche Regionen zu fördern, die ein integriertes REK mit regionalen Leitbildern und Leitprojekten vorweisen können.

Das REK für die Region Flensburg/Schleswig umfaßt den Kreis Schleswig-Flensburg und die kreisfreie Stadt Flensburg (3). Zu seinen zentralen Zielen gehört, die Identität der Region nach innen und ihre Außendarstellung zu stärken. Für eine Zusammenführung der verschiedenen Interessen mußten an den innerregionalen räumlichen und fachlichen Schnittstellen Integrationsleistungen erbracht werden. Mit Blick auf eine Selbstorganisation der Akteure nahm das IÖW die thematische Schwerpunktsetzung in einem ergebnisoffenen Konsultationsprozess vor. Neben der wissenschaftlichen Fundierung und methodischen Absicherung des Prozesses waren die Organisation von Kommunikation zwischen den Beteiligten und die umsetzungsorientierte Moderation von Entscheidungsprozessen seine wesentlichen Aufgaben.

\section{Die Region \\ Flensburg/Schleswig}

Die Region Flensburg/Schleswig liegt im Norden Schleswig-Holsteins an der 0stsee und an der Grenze zu Dänemark. Es handelt es sich um eine dünn besiedelte, landwirtschaftlich geprägte und landschaftlich reizvolle Region von hoher Umweltqualität. Sie steht infolge der veränderten politischen und ökonomischen Bedingungen in Deutschland und Europa vor besonderen Anpassungserfordernissen. Einige Bereiche mit überdurchschnittlicher Bedeutung für die regionale Wertschöpfung und Beschäftigung, so die Landwirtschaft, das Ernährungsgewerbe, der Tourismus und der öffentliche Sektor, befinden sich unter starkem Druck. Der Rückgang im Schiffbau und in der hafenabhängigen Wirtschaft verschärft die strukturellen Probleme. Positive Entwicklungen finden vor allem bei produktionsnahen Dienstleistungen und im Bereich innovativer Technologien statt. Aus den landfesten Verkehrswegen, die die Region zukünftig an den skandinavischen Wirtschaftsraum anbinden, ergeben sich neue Chancen. Die Kapazitäten und Lehrangebote der Hochschulen insbesondere in wirtschaftsnahen Studiengängen wurden in den letzten Jahren deutlich ausgebaut. Von einigen großen Produktionsbetrieben gehen kräftige Impulse für die wirtschaftliche Entwicklung der ganzen Region aus. Dennoch liegen die Arbeitslosenquoten insbesondere in Flensburg in alarmierender Höhe und die Einkommen der privaten Haushalte unter dem Bundesdurchschnitt. Bei einer wachsenden Anzahl an Langzeitarbeitslosen ist die Sozialhilfebedürftigkeit entsprechend hoch.

In dieser Ausgangslage leiteten die Akteure Entwicklungsziele $\mathrm{ab}$ und identifizierten regionale Potentiale. Sie legten querschnittsorientierte Handlungsfelder fest und erarbeiteten gemeinsam mit dem IöW Strategien für die weitere Entwicklung der Region (siehe Abb. 1, S. 32).

\section{Neve Bündnisse}

Eine Reihe von Akteuren nutzte die Gelegenheit, auf der neu entstandenen regionalen Plattform Bündnispartner ausfindig zu machen. Sie erarbeiteten innovative Projektideen oder brachten andere schon vorhandene Ideen, die inhaltlich mit den Zielen einer nachhaltigen Regionalentwicklung übereinstimmten, voran. So entwickelten deutsche und dänische Akteure aus Tourismus, Landwirtschaft und Kultur gemeinsam ein grenzuibergreifendes Projekt für die Zeit der Rapsblïte, das die nebensaisonale Unterauslastung des Tourismus beheben helfen soll. Hierfür wurde noch im Rahmen des REK ein Förderantrag gestellt, der inzwischen bewilligt ist. Eine andere Gruppe, die sich aus Vertreterinnen und Vertretern der sechs regionalen Wissens- und Technologietransferanbieter zusammensetzt, intensivierte ihre vorausgegangene Zusammenarbeit und wird noch in diesem Jahr einen gemeinsamen Flyer über die verschiedenen Transfer- und Beratungsangebote herausgeben.

\section{Umsetzung des REK absichern}

Regionalpolitik berïhrt zentrale Felder der ökonomischen, ökologischen und sozialen Entwicklung. Ihre integrierte Behandlung setzt ressortiibergreifende konsensuale Zielorientierungen und einen abgestimmten Einsatz von Problemlösungspotentialen voraus. In der Region Flensburg/Schleswig ist mit der Grïndung der Wirtschaftsförderungs- und Regionalentwicklungsgesellschaft und einer grenzüber- 


\begin{tabular}{|c|c|}
\hline Handlungsfeld & zentrale Sirategien \\
\hline $\begin{array}{l}\text { Innovation und Kooperation } \\
\text { zwischen Wirtschaft und } \\
\text { Wissenschaft }\end{array}$ & $\begin{array}{l}\text { - gemeinsame Entwicklung und Vermarktung eines regionalen Standortprofils } \\
\text { durch die regionalen Transferanbieter } \\
\text { regionale Innovationsförderung durch spezialisierte, integrierte Beratungsange- } \\
\text { bote an die Unternehmen }\end{array}$ \\
\hline Brücke des Nordens & $\begin{array}{l}\text { Erweiterung der deutsch-dänischen Infrastruktur um skandinavische leistungs- } \\
\text { elemente } \\
\text { - Nutzung der neuen landfesten Verkehrsverbindungen nach Skondinavien } \\
\text { Förderung des wissenschaftlichen und kulturellen Austausches im Ostseeroum }\end{array}$ \\
\hline Freizeit, Tourismus und Kultur & $\begin{array}{l}\text { - kulturelle Profilierung des Tourismus und Schärfung des wassertouristischen Profils } \\
\text { - Effektivierung des Managements } \\
\text { - Durchführung saisonal angepaßter Qualifizierungsmaßnahmen } \\
\text { - Entwicklung innovativer elektronischer Tourismusdienstleistungen }\end{array}$ \\
\hline Landwirtschaft/Enährung & $\begin{array}{l}\text { durchgängige Qualitätssicherung insbesondere im Fleischbereich } \\
\text { Entwicklung von Einkommenskombinationen und -alternativen etwa in Bereich } \\
\text { der Regionalvermarkiung } \\
\text { - Ausbau der innerregionalen Lieferverflechtungen und der Weiterverarbeitungs· } \\
\text { einrichtungen } \\
\text { - Förderung des ökologischen Landbaus }\end{array}$ \\
\hline $\begin{array}{l}\text { Verkehrsinfrastruktur und } \\
\text { Transportwirtschaft }\end{array}$ & $\begin{array}{l}\text { - Fortentwicklung und Ausbruu der Logistik-Dienstleistungen } \\
\text { - Verknüpfung der vorhandenen logistischen Komponenten in einem grenz. } \\
\text { übergreifenden Güterverkehrskonzept } \\
\text { - Beteiligung an der Planung überregionaler Verkehrsanbindungen }\end{array}$ \\
\hline $\begin{array}{l}\text { Gewerbeflöchenentwicklung } \\
\text { und weiche Infrostrukturen }\end{array}$ & $\begin{array}{l}\text { Erstellung eines innovationsorientierten regionalen Siedlungsstrukiur-und } \\
\text { Freiraumkonzeptes } \\
\text { qualitative Angebote an KMWU im Bereich hochrechnologischer Produkte und an } \\
\text { den gesamten Bereich der wirtschaftsnahen Dienstleister }\end{array}$ \\
\hline
\end{tabular}

Quelle: eigene Darstellung

schreitenden Koordinierungsstelle bereits für eine Zusammenfiihrung verschiedener Bereiche gesorgt. Dennoch ist abzusehen, daß eine Umsetzung des REK mit den bestehenden Steuerungs- und Koordinierungskapazitäten nicht geleistet werden kann. Das IÖW hat daher dringend zu einer institutionellen Absicherung der erforderlichen Arbeitsschritte geraten und der Politik empfohlen, eine Stelle für regionales Management zu schaffen. Zu deren Aufgaben würden die Begleitung regionaler Projekte, ihre Unterstïtzung unter Berücksichtigung von Wechselwirkungen und Synergieeffekten, die Koordination ressortübergreifender projektbezogener Kooperation, die Vereinheitlichung der Planungen zwischen den Gebietskörperschaften und der konstruktive Dialog mit der regionalen Politik gehören.

Eine solche Koordinierungsstelle ist auch notwendig, damit in der weiteren Entwicklung dem eingebrachten Engagement der Akteure poliallem sicherzustellen, daß das REK der Bevölkerung zugänglich gemacht und unter Mithilfe der regionalen Medien öffentlich zur Diskussion gestellt wird. Dies ist die Voraussetzung dafuir, daß die regionalen Handlungsträger sich in ihrer täglichen Arbeit auf das REK berufen und die formulierten Ansprüche einlösen können. Erst die Überführung der initiierten Entwicklungen in stabile, kontinuierlich tragfähige Strukturen der Zusammenarbeit macht das REK zu einem zuverlässig wirksamen Politikinstrument. Der Verzicht darauf würde den angestoßenen Prozeß der Demokratisierung und wachsenden Selbstverantwortung konterkarieren und die durch das IÖW ausgeräumte Skepsis mancher Akteure gegeniiber ,noch einem Gutachten“ erneut bestärken.
Sinnvoll ist, an den dialogorientierten, sektorübergreifenden Arbeitsformen anzuknüpfen, die kreative Potentiale freigesetzt und neue Kooperationen ermöglicht haben. Im Verlauf des REK sind bereits Impulse und Initiativen entstanden, die die Region im Wettbewerb „Regionen der Zukunft auf dem Weg zu einer nachhaltigen Entwicklung" gestärkt haben: in dem von der Bundesanstalt für Bauwesen und Raumordnung durchgefïhrten Wettbewerb zeichnete das Bundesministerium für Verkehr, Bau- und Wohnungswesen im Juni 1998 die Region Flensburg/Schleswig als ,Zukunftsregion" aus.

\section{Anmerkungen}

(1) Das Regionale Entwicklungskonzept wurde unter Leitung von Rainer Lucas von den IÖW-Forschungsfeldern Regionale Wirtschaftspolitik/ Nachhaltige Regionalentwicklung (Wuppertal) und Stadtentwicklung, Planung, Verkehr (Berlin) erarbeitet.

Vgl. Lucas, R./ H. Flämig/ G. Nischwitz/ G.E. Stevens/ B. Weißner/S. Wittek: Regionales Entwicklungskonzept für die Region Flensburg/Schleswig, Abschlußbericht. Erstellt im Auftrag der Wirtschaftsförderungs- und Regionalentwicklungsgesellschaft WiREG mbH Flensburg/Schleswig. Wuppertal, 1999. Als CD-ROM zu beziehen bei der WiREG mbH, E-mail: info@wireg.de. Der Bericht wird demnächst auch als IÖW - Schriftenreihe erscheinen.

(2) Das Ministerium für Wirtschaft, Verkehr, Technologie und Verkehr des Landes. sowie die Landesplanung unterstützten das Vorhaben. Die Geschäftsstelle des Regionalprogramms übernahm die fachliche Begleitung. Zur kontinuierlichen Betreuung der Arbeit wurde eine Steuerungsgruppe gebildet, der die leitenden Persönlichkeiten aus Politik, Verwaltung und Wirtschaft der beteiligten Gebietskörperschaften angehörten.

(3) Der Kreis Nordfriesland und das Amt Sonderiylland (Dänemark) wurden in den Prozeß einbezogen. 
(c) 20I0 Authors; licensee IÖW and oekom verlag. This is an article distributed under the terms of the Creative Commons Attribution Non-Commercial No Derivates License (http://creativecommons.org/licenses/by-nc-nd/3.o/), which permits unrestricted use, distribution, and reproduction in any medium, provided the original work is properly cited. 